\title{
Mesoporous Gold Sponge as a Prototype 'Meta-material'
}

\author{
Abbas I. Maaroof, Michael B. Cortie, Angus Gentle, Geoffrey B. Smith \\ University of Technology Sydney, Sydney, NSW, Australia, \\ geoff.smith@uts.edu.au
}

\begin{abstract}
Mesoporous gold sponge has optical properties that can be intermediate between those of metals and insulators, with a flat spectral response that is unlike that of bulk gold. Films of different thicknesses were produced and an extension of the Lorentz-Drude (LD) model used to model their spectral behavior. We found that it was necessary to include an additional special oscillator centered at $1.4 \mathrm{eV}$ in order to model the unusual spectral response. This is quite unlike bulk gold, which can be modeled using a standard two-oscillator LD model.
\end{abstract}

Keywords: mesoporous, optical properties, DC magnetron sputtering, gold thin films

\section{Introduction}

Conducting metamaterials which are highly porous and mechanically stable can be prepared by making certain thin film binary alloy combinations followed by selective chemical etching of one component [1-3]. To produce mesoporous gold the pre-cursor coating we use is $\mathrm{AuAl}_{2}$ from which the aluminium is removed by etching. During the removal of aluminium the gold metal atoms rearrange $[3,4]$ to form a stable percolating network of gold plus many irregularly shaped pores. The gold content in all of the mesoporous films we have produced to date is in the minority in volume fraction terms, typically in the range $35 \%$ to $10 \%$ and surface areas can be very high at $\sim 2 \mathrm{~m}^{2} / \mathrm{g}$. Their average or homogenised dc conductivity is good, being in the range $1 \times 10^{5}$ to $3 \times 10^{6}(\Omega \mathrm{m})^{-1}$ compared with that of dense Au films of around $4 \times 10^{7}(\Omega \mathrm{m})^{-1}$. This is despite the twin impacts of geometry (the high porosity and the associated tortuous conducting paths), and the significantly increased relaxation rate of conducting electrons in the nanostructured gold.

The pores have transverse dimensions in the range of only 10 to $30 \mathrm{~nm}$ so the gold/void metamaterial behaves optically as if it is homogeneous and is thus a candidate for modelling with quasi-static effective medium theories. The experimental effective optical properties, thermal emittance and void content of the resulting mesoporous gold are sensitive to the conditions used during deposition of the pre-cursor $\mathrm{AuAl}_{2}$ thin film. Variations in such factors as temperature of the substrate, rate of sputter deposition, use of ion assistance, and deposition pressure for the pre-cursor film can lead to significant alterations in the levels of porosity and gold carrier relaxation rates. At first glance the mesoporous layers appear to be neither metallic nor to act as classical dielectrics since they have unusually flat spectral response in the near IR [1]. However, it is known that nanoscale structure can lead in some instances to additional electronic states in the gold, an issue of great importance in respect of the use of this material in chemical catalysis [5]. We will show that there is evidence for this here too and that the material has additional electronic states that manifest optically via low energy transitions, centred at $1.4 \mathrm{eV}$. These states do not, as far as we can tell using effective medium treatments and studies of different thicknesses, emerge from resonances induced inside or on the mesostructure. They have a strong influence on near infra-red optical response of the metamaterial by delaying the onset of dominance of the Drude term and hence of strong plasmonic behaviour. In this paper we focus on samples showing such novel behaviour from the perspective of their effective dielectric response.

\section{Experimental details}

\subsection{Film fabrication}

The fabrication process of the mesoporous gold films is similar to that described previously $[1,2]$. Briefly, alloy films of $\mathrm{AuAl}_{2}$ were prepared by co-depositing the elements using high vacuum dc 
magnetron sputtering onto glass substrates. To ensure good homogeneity, uniformity and crystallinity, a rotating stage substrate set at $400^{\circ} \mathrm{C}$ temperature was used during deposition. After co-depositing of $\mathrm{AuAl}_{2}$ on a glass substrate, aluminium was removed from the compound by immersing the films in $\mathrm{NaOH}(0.2 \mathrm{M})$ solution.

\subsection{Characterisation}

Scanning electron microscopy (SEM) with a Zeiss Supra 55VP was used to study the nanostructure of the films. Figure 1 shows a typical SEM image one of the mesoporous gold films (thickness $=37.5 \mathrm{~nm}$ ) that displayed unusual NIR properties. Spectral transmittance $T(\lambda)$ and reflectance $R(\lambda)$ of the mesoporous gold films were carried out using a Perkin-Elmer Lambda 950 UV/VIS/NIR spectrophotometer and normal incidence over the wavelength range $300 \mathrm{~nm}<\lambda \square<2500 \mathrm{~nm}$. Ellipsometry data taken at high incidence angles enabled a check of complex optical constants $(\mathrm{n}, \mathrm{k})$ found from spectrophotometry, and assessment of optical isotropy. The optical constants $n(\lambda)$, and $\mathrm{k}(\lambda)$ were determined from $\mathrm{T}(\lambda)$ and $\mathrm{R}(\lambda)$ and approximate prior measurements of thickness using the best fit single layer models given by the WVASE32 software from J. A. Woollam Co., Inc.

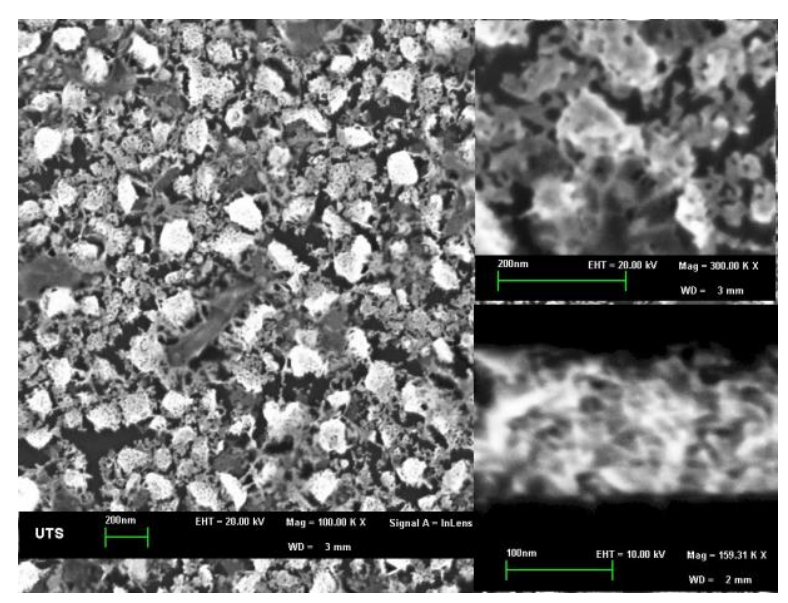

Figure 1: Mesoporous gold film of $38 \mathrm{~nm}$ thickness on glass, which displays optical transitions near $1.4 \mathrm{eV}$. Top right inset shows a high magnification view of surface, bottom right inset shows crosssection view of the film.

\section{Results and discussion}

\subsection{Lorentz-Drude model (Special triple-oscillator)}

The experimental $T(\lambda)$ and $R(\lambda)$ spectra shown in figure 2 for all the samples display a response which is qualitatively not like normal metals, nor like dielectrics. The classical approach for the frequency-dependent dielectric function $\varepsilon(\omega)$ of dense gold films is the standard Lorentz-Drude (LD) model. The LD model can also be used to model mesoporous gold but that it has to be extended to cover changes in electronic parameters. The general LD model for $\varepsilon(\omega)$ is expressed as [6]

$$
\hat{\varepsilon}(\omega) \equiv \varepsilon_{1}+\mathbf{i} \varepsilon_{2}=\varepsilon_{\infty}+\sum_{\mathbf{k}} \frac{\mathbf{C}_{\mathbf{k}}}{\omega_{\mathbf{k}}^{2}-\omega\left(\omega+\mathbf{i} \omega_{\tau, \mathbf{k}}\right)},
$$

where $\varepsilon_{\infty}$ is a constant, above 1 , due to other higher energy interband (ib) transitions in the UV, $C_{k}$ is the amplitude of each oscillator or its spectral weight, $\omega_{\mathrm{k}}$ is the centre energy, and, $\omega_{\tau, \mathrm{K}}$ is the broadening of each oscillator. 

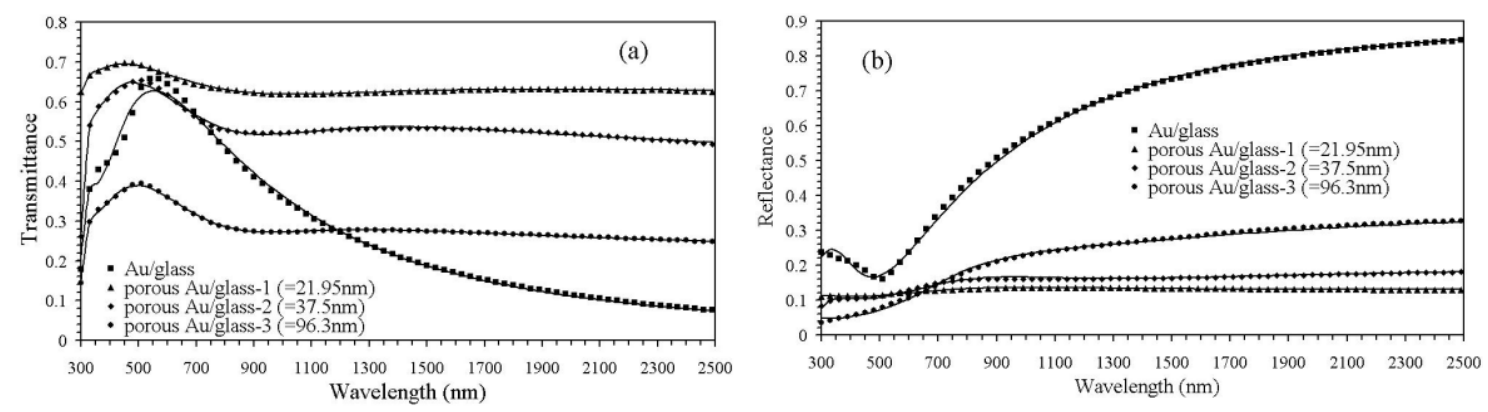

Figure 2: (a) Spectral transmittance and (b) spectral reflectance experimental data for three mesoporous gold films with solid lines representing the fitted models (special L-D model for the mesoporous films and standard L-D model for the $10.7 \mathrm{~nm}$ dense gold film).

We used two oscillators for the dense gold film $(10.7 \mathrm{~nm})$ of figure 2 because we had to consider the influence of the interband transitions in the visible region (where $\mathrm{k}=1$ ), as well as the contribution of the free electrons (Drude model, where $\mathrm{k}=0$ and $\omega_{\mathrm{k}}=0$ ). The data for the dense gold film was readily fitted using equation (1) with two oscillators. The LD parameters are provided in table 1 . However mesoporous gold with its peculiar flat spectral response could only be fitted by inclusion of a third oscillator located in the near-infrared (NIR) region. The resulting model becomes.

$\hat{\varepsilon}(\omega)=\varepsilon_{\infty}+\left[\frac{C_{i b}}{\omega_{i b}^{2}-\omega\left(\omega+i \omega_{\tau, \mathrm{ib}}\right)}\right]+\left[\frac{C_{\mathrm{NIR}}}{\omega_{\mathrm{NIR}}^{2}-\omega\left(\omega+\mathrm{i} \omega_{\tau, \mathrm{NIR}}\right)}\right]-\left[\frac{\omega_{\mathrm{p}}^{2}}{\omega\left(\omega+\mathrm{i} \omega_{\tau}\right)}\right]$

which provided a far better fit as shown in figure 3.
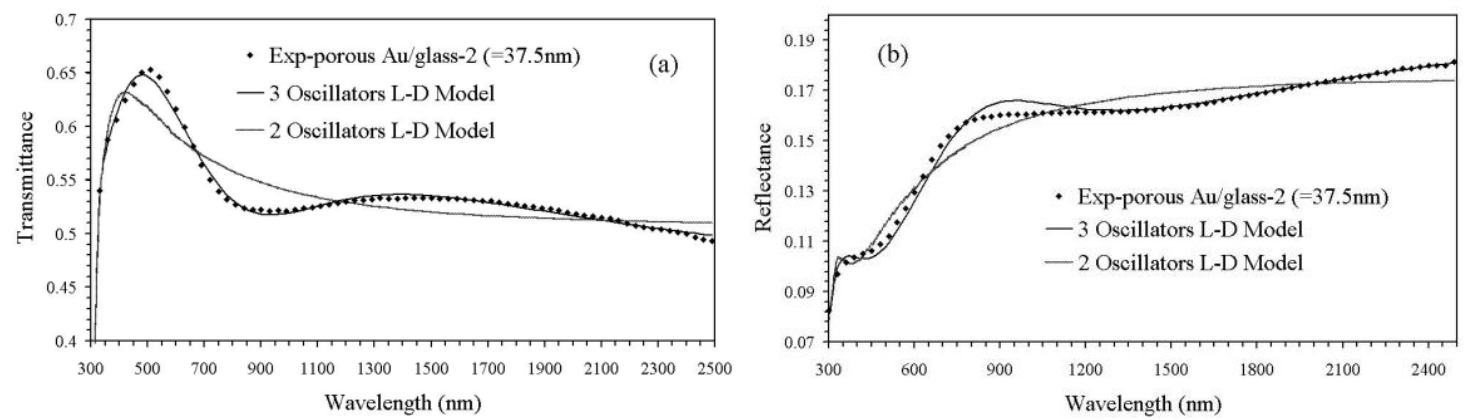

Figure 3: a) Spectral transmittance and (b) spectral reflectance experimental data for one of three mesoporous gold films (sample at thickness $=37.5 \mathrm{~nm}$ ), fitted with the standard two oscillators LD model (poor fit) and special triple oscillator LD model (good fit).

\subsection{The new gold state and its origins?}

The term of main interest here is the NIR oscillator, the second term in equation (2). The central energy is at $1.4 \mathrm{eV}$ in all samples, and the two thicker samples have a similar spectral weight of $\sim 3$ $\mathrm{eV}^{2}$ and bandwidth of $\sim 1.35 \mathrm{eV}$. The contribution from this source is of similar magnitude to the normal interband term so there are a lot of these new states present. The physical origins of these transitions have not as yet been identified and further study will be needed to clarify their source. However, our hypothesis is that the rough, mesoporous surfaces contain an abundance of surface gold atoms of low coordination which could be responsible by virtue of them having subtly different electronic states to the bulk. (This is also believed to be the explanation for the catalytic properties of gold nanoparticles.) Other possibilities can be postulated such as surface states, which arise when the gold atoms have greater freedom to migrate in very open structures produced by etching. Novel quantum states near the Fermi surface associated with strongly correlated pairing of d electrons as 
occurs in $\mathrm{VO}_{2}$ [12] cannot be ruled out either. In metallic $\mathrm{VO}_{2}$ these states lead to the requirement for an LD oscillator with optical transitions near $1 \mathrm{eV}$, which significantly suppresses the strength of near infrared plasmonic responses.

Table 1: Fitted parameters for the L-D model including an NIR oscillator for the three mesoporous gold films of thickness $22.0 \mathrm{~nm}, 37.5 \mathrm{~nm}$ and $96.3 \mathrm{~nm}$. The samples were made using the same deposition and etching conditions to see if effective optical response altered significantly with growth.

\begin{tabular}{|c|c|c|c|c|c|c|c|c|c|}
\hline \multirow[b]{2}{*}{$\begin{array}{l}\text { Samples } \\
\text { and } \\
\text { thicknesses }\end{array}$} & \multirow{2}{*}{$\varepsilon_{\infty}$} & \multicolumn{2}{|c|}{$\begin{array}{l}\text { Drude term } \\
\text { (Oscillator 1) }\end{array}$} & \multicolumn{3}{|c|}{$\begin{array}{l}\text { NIR term } \\
\text { (Oscillator 2) }\end{array}$} & \multicolumn{3}{|c|}{$\begin{array}{l}\text { Interband term } \\
\text { (Oscillator 3) }\end{array}$} \\
\hline & & $\begin{array}{r}\omega_{\mathrm{p}}{ }^{*} \\
(\mathrm{eV})\end{array}$ & $\begin{array}{c}\omega_{\tau, \text { meso }} \\
(\mathrm{eV})\end{array}$ & $\begin{array}{l}\mathrm{C}^{*}{ }_{\mathrm{NIR}} \\
\left(\mathrm{eV}^{2}\right)\end{array}$ & $\begin{array}{l}\omega_{\tau, \mathrm{NIR}} \\
(\mathrm{eV})\end{array}$ & $\begin{array}{l}\omega_{\mathrm{NIR}} \\
(\mathrm{eV})\end{array}$ & $\begin{array}{l}\mathrm{C}^{*}{ }_{\mathrm{Au}} \\
\left(\mathrm{eV}^{2}\right)\end{array}$ & $\begin{array}{c}\omega_{\tau, \mathrm{Au}, \mathrm{ib}} \\
(\mathrm{eV})\end{array}$ & $\begin{array}{l}\omega_{\text {Au.ib }} \\
(\mathrm{eV})\end{array}$ \\
\hline $\begin{array}{l}\text { Dense Au } \\
(10.7 \mathrm{~nm})\end{array}$ & 4.6 & 8.6 & 0.09 & - & - & - & 41.0 & 1.9 & 3.5 \\
\hline \begin{tabular}{|l} 
Porous Au 1 \\
$(21.95 \mathrm{~nm})$
\end{tabular} & 1.7 & 2.1 & 0.80 & 6.2 & 2.0 & 1.4 & 7.9 & 2.8 & 3.9 \\
\hline $\begin{array}{l}\text { Porous Au } 2 \\
(37.5 \mathrm{~nm})\end{array}$ & 2.0 & 2.2 & 0.86 & 3.3 & 1.3 & 1.4 & 7.1 & 2.4 & 3.9 \\
\hline $\begin{array}{l}\text { Porous Au } 3 \\
(96.3 \mathrm{~nm})\end{array}$ & 2.8 & 2.4 & 1.05 & 2.6 & 1.4 & 1.4 & 8.8 & 3.2 & 3.8 \\
\hline
\end{tabular}

\section{Conclusions}

Very thin mesoporous gold spongy films of different thicknesses on glass substrate have been produced and all of them show the same spectral responses, being neither metal -nor insulator-like. Application of a generalised Lorentz-Drude model revealed that all the mesoporous gold samples required an additional special oscillator at low energy centred at $1.4 \mathrm{eV}$ in order for a reasonable fit to be obtained. This is quite unlike dense gold film. The mesoporous structure is in effect behaving as a new material.

\section{Acknowledgement}

The authors thank the University of Technology Sydney, Australia, for support.

\section{References}

1. A. I. Maaroof, M. B. Cortie and G. B. Smith, J. Optics A, Pure and Applied Optics 7, 303-309 (2005).

2. 2. M. B. Cortie, A. Maaroof, and G. B. Smith, P. Ngoepe, Current Applied Physics, 6, 440-443 (2006).

3. J. Erlebacher, M.J. Aziz, A. Karma, N. Dimitrov and K. Sieradzki, Nature 410, 450 -453 (2001)

4. J. Erlebacher, J. Electrochem. Soc., 151, C614-C626 (2004)

5. M. Valden., X. Lai, and D.W. Goodman : Science, vol.281, pp.1647-1650 (1998)

6. G.B. Smith, A.I Maaroof, A.R. Gentle and M.B. Cortie Proc SPIE in press Proceedings of Photonics Europe 2006 (Photonics for Solar Energy Systems) Vol 6197 paper 29

8. S. Biermann, A. Poteryaev, A.I. Lichtenstein and A. Georges Phys. Rev. Lett., 94, 026404-1-3, (2005) 